\title{
Utilisation du quadrangle « discrete shear" pour la génération des surfaces d'influence des efforts de plaque
}

\author{
Exemples de mise en œuvre avec Code_Aster
}

\author{
Philippe Lévêque - Florent Bacchus \\ Service d'études techniques des routes et des autoroutes \\ Centre des Techniques d'Ouvrages d'Art, Division des Logiciels et Ouvrages Types \\ 46 avenue Aristide Briand \\ F-92229 Bagneux cedex \\ \{philippe.leveque, florent.bacchus\}@equipement.gouv.fr
}

\begin{abstract}
RÉSUMÉ. La notion de surface d'influence constitue l'outil approprié pour déterminer les effets structuraux extrêmes dans les structures soumises à l'action de charges mobiles. C'est ainsi que l'on calcule l'effet du trafic routier s'exerçant sur les tabliers des ponts. La méthode de génération des surfaces d'influence s'appuie sur le théorème de réciprocité de MaxwellBetti. Cet article présente l'utilisation de la formulation "discrete shear " d'élément fini quadrangle de plaque épaisse pour générer les surfaces d'influence des efforts internes dans un pont-dalle à l'aide du logiciel Code_Aster Libre.

ABSTRACT. Concept of influence surface is the appropriate tool to determine the extreme structural response values of structures under the effect of moving loads. The action of road traffic moving on bridge deck is computed this way. Influence surface generation method is based on Maxwell-Betti's theorem of reciprocity. This paper presents the use of the "discrete shear " formulation of a thick plate quadrangle finite element to generate the influence surfaces of internal forces in a slab bridge using the Code_Aster free software.

MOTS-CLÉS : surface d'influence, effets structuraux, charges mobiles, théorème de réciprocité de Maxwell-Betti, pont-dalle, élément fini, plaque épaisse, formulation discrete shear, Code_Aster Libre.

KEYWORDS: influence surface, structural responses, moving loads, Maxwell-Betti's theorem of reciprocity, slab bridge, finite element, thick plate, discrete shear formulation, Code_Aster free software.
\end{abstract}

DOI:10.3166/REMN.17.495-527 @ 2008 Lavoisier, Paris 


\section{Introduction}

Le calcul des effets structuraux dans les ponts présente une spécificité qui tient au fait que ces structures sont soumises, au droit de leur tablier, à des charges mobiles, à savoir le trafic routier. Le projeteur doit déterminer la distribution (nombre et positionnement) des charges de trafic conduisant aux effets structuraux extrêmes dans la structure. Cette recherche peut s'effectuer de façon simple en s'appuyant sur la notion d'influence.

Bien connue et largement utilisée dans le cas des modélisations filaires des structures, cette notion reste peu utilisée dans le cas des modélisations surfaciques ou volumiques.

Le présent article a pour objectifs de présenter la méthode usuelle de calcul des influences dans le cadre de l'analyse matricielle des structures, de façon générale et indépendamment du type de modélisation choisie, puis de l'illustrer dans le cas particulier des influences d'efforts internes dans les modélisations de plaques par éléments finis. La méthode est ensuite mise en œuvre sur deux cas d'étude de pontsdalle, en s'appuyant sur la formulation de l'élément fini de plaque « Discrete Shear Quadrangle» (Batoz, 1990) et sur l'utilisation du logiciel Code_Aster Libre.

\section{Notion de surface d'influence et application au calcul des ponts}

\subsection{Définitions}

Fixons-nous :

1. un point d'étude $P_{e}$ de la structure (non nécessairement sur le tablier),

2. une action mécanique $A$ (force verticale, force horizontale, couple...),

3. un effet mécanique $E$ (déplacement, déformation, contrainte, effort...),

puis associons à un point du tablier, appelé point de charge $\left(P_{c}\right)$, la valeur $E^{A}\left(P_{e}, P_{c}\right)$ de $E$ en $P_{e}$ lorsqu'on exerce une action $A$ unité en $P_{c}$. On dit que $E^{A}\left(P_{e}, P_{c}\right)$ représente l'influence de l'action unitaire $A$ exercée au point $P_{c}$ sur l'effet $E$ au point $P_{e}$.

Le résultat de ce processus lorsque $P_{c}$ parcourt le tablier est l'obtention d'une fonction d'influence $P_{c} \rightarrow E^{A}\left(P_{e}, P_{c}\right)$ qui peut être représentée graphiquement :

- dans le cas d'une modélisation filaire, on parle de ligne d'influence,

- dans le cas d'une modélisation surfacique, on parle de surface d'influence. 


\subsection{Application}

Le trafic routier applicable aux tabliers de ponts est défini règlementairement : en France, le Fascicule 61 Titre II a longtemps été appliqué avant de céder sa place à l'actuelle réglementation européenne (Eurocode 1). De manière générale, le trafic routier règlementaire est constitué :

- d'un ensemble de charges concentrées (forces verticales $F_{i}$ appliquées en des points $P_{i}$ du tablier),

- d'une charge répartie (force verticale $f$ appliquée sur une zone $\omega$ du tablier),

de sorte qu'en analyse linéaire, l'effet au point $P_{e}$ d'une distribution de trafic routier est fourni par la formule [1] :

$$
\sum_{i} F_{i} E^{A}\left(P_{e}, P_{i}\right)+\int_{\omega} f(P) E^{A}\left(P_{e}, P\right) d \omega(P)
$$

où $A$ représente ici une force verticale.

Il s'agit pour le projeteur de déterminer pour chaque point d'étude $P_{e}$, parmi l'ensemble des charges autorisées règlementairement, les charges routières conduisant aux effets structuraux extrêmes en $P_{e}$. Les points d'étude résultent en général d'une discrétisation régulière de la structure, mais leur densité peut parfois être renforcée au droit de zones particulières (angles...).

Lorsqu'il dispose d'une représentation graphique des influences (ligne ou surface d'influence), le projeteur est donc guidé dans sa recherche des effets extrêmaux : il positionne les charges routières au droit des zones du tablier où la valeur absolue de la fonction d'influence est forte ou faible selon le caractère favorable ou défavorable recherché.

Les surfaces d'influence étaient traditionnellement fournies au projeteur sous forme d'abaques, par exemple (Rüsh, 1964). Aujourd'hui, les moyens informatiques permettent au projeteur de générer lui-même numériquement les surfaces adaptées à la configuration qu'il étudie, notamment par l'emploi d'un logiciel de calcul aux éléments finis. Cependant, cette solution reste peu utilisée, alors que la génération numérique des lignes d'influence est classique, principalement pour deux raisons :

- d'une part, l'emploi de méthodes de calcul simplifiées (i.e. utilisant des modélisations filaires) pour certaines structures relevant pourtant a priori d'une modélisation surfacique (par exemple, les ponts-dalle), reste encore très répandu parmi les projeteurs. Bien souvent en effet, l'emploi de telles méthodes est suffisant. Cependant, dans certaines situations, (par exemple, les ponts-dalle présentant un biais géométrique prononcé), le projeteur ne peut pas faire l'économie d'une modélisation surfacique,

- d'autre part, l'exploitation rigoureuse des influences (recherche systématique des effets extrêmaux et des distributions de trafic correspondantes) est moins complexe à mettre en œuvre dans le cas filaire que dans le cas surfacique, pour lequel il est pratiquement indispensable de recourir à des outils dédiés. De tels 
logiciels ont notamment été développés par le Sétra, service du ministère de l'écologie, du développement et de l'aménagement durable, en tant qu'outils internes non diffusés. Les outils commerciaux possédant ces fonctionnalités sont encore peu développés en France. Au niveau international, certains éditeurs proposent des logiciels dont les pages web annoncent ce genre de fonctionnalités. Citons, sans avoir une connaissance précise des fonctionnalités effectivement implémentées, ni sans prétendre à l'exhaustivité, les logiciels suivants : LARSA4D (http://www.larsa4d.com), LUSAS Bridge (http://www.lusas.com), MIDAS/Civil (http://www.cspfea.net), SAP2000 (http://www.csiberkeley.com/products_SAP. html), Smartbridge (http://www.smartbridgetech.com), STAAD.Pro (http://www. reiworld.com), SuperSTRESS (http://www.integer-software.co.uk/engineeringsoftware), TDV (http://www.tdv.at).

La technique de calcul numérique des surfaces d'influence est classiquement fondée sur la notion de dualité et sur le principe de réciprocité de Maxwell-Betti. Plutôt que d'obtenir les surfaces d'influence en imposant successivement en chaque point du tablier une action unité et en relevant à chaque calcul la valeur de l'effet en $P_{e}$, ce principe permet de déterminer un chargement « fictif » générant directement les influences recherchées en un seul calcul. Déjà, Jenssen (1941) met en œuvre cette technique pour l'étude, par la méthode des différences finies, des plaques biaises en citant N. M. Newmark. Plus récemment, Orakdögen (2005) la décline dans le cadre général de la méthode des éléments finis (plaques, poutres...), en ayant recours à la formulation des éléments en question. Enfin, des exemples de mise en œuvre par éléments finis (surfaces d'influence de moments dans les plaques) ont été fournis par Le Faucheur (2005) et Brisard (2007).

\section{Calcul des influences par les méthodes matricielles d'analyse des structures}

\subsection{Principe général}

\subsubsection{Généralités et notations}

L'approche générale poursuivie par les méthodes matricielles d'analyse des structures reste la même indépendamment du type de structure considérée (solide tridimensionnel, poutre, plaque, coque...). Il s'agit de représenter la structure comme un assemblage d'éléments structuraux reliés les uns aux autres en un nombre fini de points nodaux. La connaissance des relations forces-déplacements pour chaque élément permet d'en déduire le comportement de la structure assemblée (Zienkiewicz, 1973). L'analyse classique des réseaux de poutres relève de cette approche. La méthode des éléments finis relève également de cette approche. Elle constitue en quelque sorte une extension au milieu continu des techniques matricielles mises en œuvre pour les réseaux de poutres. 
Considérons donc ici que la structure est constituée d'éléments reliés entre eux par des nœuds. On fait ainsi intervenir :

- un vecteur $\{e\}$ d'effets nodaux (en général des déplacements, éventuellement des déplacements dits « généralisés » tels que des rotations),

- un vecteur $\{a\}$ d'actions nodales (en général des forces, éventuellement des forces dites « généralisées » telles que des couples).

Dans le cas du calcul des structures par la méthode dite «des forces », on est amené à résoudre le système $\{e\}=[S]\{a\}$, d'inconnue $\{a\}$, où $[S]$ est la matrice de souplesse. Par contre, dans le cas de la méthode dite «des déplacements ", l'inconnue est le vecteur $\{e\}$ et on doit résoudre le système $\{a|=| K \mid\{e \mid$, où $[K]$ est la matrice de rigidité.

Remarque 1. - La formulation de la méthode des éléments finis couramment utilisée par les ingénieurs en calcul de structure relève de la méthode des déplacements.

Remarque 2. - Les matrices $[S]$ et $[K]$ résultant de la modélisation du problème sont symétriques.

Considérons que le maillage de la structure comporte $m$ nœuds et qu'en chaque nœud, on ait $n$ composantes d'action (et autant de composantes d'effet). Notons alors :

- $\left\{e^{i}\right\}$ le vecteur, de dimension $n$, des effets au nœud $i$,

- $\left\{a^{j}\right\}$ le vecteur, de dimension $n$, des actions au nœud $j$,

- $e_{\gamma}^{i}$ et $a_{\gamma}^{j}$ les composantes d'indice $\gamma$ (où $1 \leqslant \gamma \leqslant n$ ) de $\left\{e^{i}\right\}$ et de $\left\{a^{j}\right\}$ : ces 2 composantes sont dites duales, au sens où l'action $a_{\gamma}^{j}$ peut «travailler» dans l'effet $e_{\gamma}^{i}$ (par exemple, une force dans une direction $\gamma$ travaille dans un déplacement de même direction) : $a_{\gamma}^{j} \cdot e_{\gamma}^{i}$ représente alors un travail :

- le vecteur $\{a\} \quad$ (resp. $\{e\} \quad$ ) sous forme de blocs $\left(\begin{array}{c}\vdots \\ \left\{a^{j}\right\} \\ \vdots\end{array}\right)$ (resp. $\left(\begin{array}{c}\vdots \\ \left\{e^{i}\right\} \\ \vdots\end{array}\right)$ )

- la matrice $[S]$ sous forme de blocs : $\left(\begin{array}{ccc}\ldots & \ldots & \ldots \\ \ldots & {\left[S^{i j}\right]} & \ldots \\ \ldots & \ldots & \ldots\end{array}\right)$

La relation $\{e\}=[S]\{a\}$ s'écrit alors : $\left(\begin{array}{c}\vdots \\ \left\{e^{i}\right\} \\ \vdots\end{array}\right)=\left(\begin{array}{ccc}\ldots & \ldots & \ldots \\ \ldots & {\left[S^{i j}\right]} & \ldots \\ \ldots & \ldots & \ldots\end{array}\right)\left(\begin{array}{c}\vdots \\ \left\{a^{j}\right\} \\ \vdots\end{array}\right)$ 


\subsubsection{Application du théorème de Maxwell-Betti au calcul des influences}

Considérons le problème P1 suivant : on fixe une composante d'action $\beta$ (avec $1 \leqslant \beta \leqslant n \quad$ ) et on s'intéresse à l'influence de cette composante appliquée au nœud $j$ sur la composante d'effet $\alpha$ (avec $1 \leqslant \alpha \leqslant n$ et éventuellement $\alpha=\beta$ ) au nœud $i$ : on applique donc $a_{\beta}^{j}=1$ et on examine $e_{\alpha}^{i}$.

La relation matricielle fournit $\left\{e^{i}\right\}=\sum_{k=1}^{m}\left[S^{i k}\right]\left\{a^{k}\right\}$, et se réduit à $\left\{e^{i}\right\}=\left[S^{i j}\right]\left\{a^{j}\right\} \quad$ (car tous les blocs $\left\{a^{k}\right\}$ sont nuls sauf $\left\{a^{j}\right\}$ ) d'où l'on tire $e_{\alpha}^{i}=\sum_{\gamma=1}^{n} S_{\alpha \gamma}^{i j} a_{\gamma}^{j}$, qui se réduit à son tour en $e_{\alpha}^{i}=S_{\alpha \beta}^{i j} a_{\beta}^{j}=S_{\alpha \beta}^{i j} \quad$ (car toutes les composantes de $\left\{a^{j}\right\}$ sont nulles sauf $a_{\beta}^{j}$ qui vaut 1 ).

On constate donc que la notion d'influence est tout à fait naturelle dans le cadre d'une méthode des forces puisque les influences correspondent aux termes de la matrice de souplesse. Dans le cas de la méthode des déplacements, les influences s'obtiennent par contre comme les termes de la matrice $[K]^{-1}$ inverse de la matrice de rigidité.

Intéressons-nous maintenant au problème $\mathrm{P} 2$ suivant (problème dit dual) : on impose une action $a_{\alpha}^{i}$ (duale de l'effet $e_{\alpha}^{i}$ ) unité, et on relève l'effet $e_{\beta}^{j}$ (dual de l'action $a_{\beta}^{j}$ ). Pour un milieu continu, le principe de réciprocité de MaxwellBetti permet d'affirmer que le travail des actions du problème P2 dans les effets du problème $\mathrm{P} 1$ est égal au travail des actions du problème $\mathrm{P} 1$ dans les effets du problème P2. Cette propriété se retrouve dans les modèles numériques fondés sur les méthodes matricielles d'analyse des structures, compte-tenu de la symétrie de la matrice $[K]$. En effet, on a la succession d'égalités [2] :

$$
\{a\}_{P 2}^{t}\{e\}_{P 1}=\{e\}_{P 2}^{t}[K]^{t}\{e\}_{P I}=\{e\}_{P 2}^{t}[K]^{t}[K]^{-1}\{a\}_{P I}=\{a\}_{P I}^{t}\{e\}_{P 2}
$$

qui conduit à l'égalité [3] :

$$
e_{\alpha}^{i}=e_{\beta}^{j}
$$

compte-tenu des chargements unitaires appliqués.

Par suite, l'ensemble des $m$ influences des actions unitaires $a_{\beta}^{j}$ sur l'effet $e_{\alpha}^{i}$ lorsque $j$ parcourt les $m$ nœuds du modèle peut s'obtenir comme les $m$ effets $e_{\beta}^{j}$ aux nœuds du modèle lorsqu'on applique l'action unitaire $a_{\alpha}^{i}$. Ainsi, au lieu d'effectuer $m$ calculs structuraux, on n'en effectue qu'un seul.

\section{Généralisation à une combinaison linéaire d'effets}

Plus généralement, on peut obtenir l'influence de $a_{\beta}^{j}$ sur une combinaison linéaire de composantes du vecteur $\{e\}$. Par exemple, l'influence sur l'effet [4] :

$$
\sum_{\gamma \in \Gamma, k \in K} c_{\gamma k} e_{\gamma}^{k}
$$


où :

$-\Gamma \subset\{1, \ldots, n\}$ et $K \subset\{1, \ldots, m\}$,

- les coefficients $c_{\gamma k}$ sont des constantes,

s'obtient en appliquant l'action duale [5] :

$$
\sum_{\gamma \in \Gamma, k \in K} c_{\gamma k} a_{\gamma}^{k}
$$

Cette généralisation se révèle particulièrement utile lorsqu'on s'intéresse à un effet qui ne figure pas parmi les composantes du vecteur $\{e\}$ mais qui peut s'exprimer linéairement en fonction de celles-ci. C'est en général le cas des contraintes et déformations dans les structures tridimensionnelles ou des contraintes et déformations généralisées dans les structures minces (poutres, plaques, coques). On donne en section 3.2 des exemples de telles relations pour les efforts (i.e. les contraintes généralisées) dans les plaques, dans le cas de la méthode des éléments finis.

\subsection{Un exemple : les surfaces d'influence des efforts dans les plaques}

\subsubsection{Rappel sur la théorie de plaque de Reissner/Mindlin}

Le modèle de plaque de Reissner est fondé sur l'hypothèse des sections droites selon laquelle les sections perpendiculaires à la surface moyenne de la plaque restent droites (planes) après déformation, mais pas nécessairement perpendiculaires à la surface moyenne déformée. Cette hypothèse conduit à une cinématique à 5 fonctions inconnues :

- les 2 déplacements $u$ et $v$ de la surface moyenne dans son plan,

- le déplacement $w$ de la surface moyenne perpendiculairement à son plan (la « flèche »),

- les 2 rotations $\beta_{x}$ et $\beta_{y}$ de la normale à la surface moyenne.

La figure 1 précise les conventions de signe employées pour les déplacements et rotations, ainsi que pour leurs actions « duales » c'est-à-dire les forces et les couples.

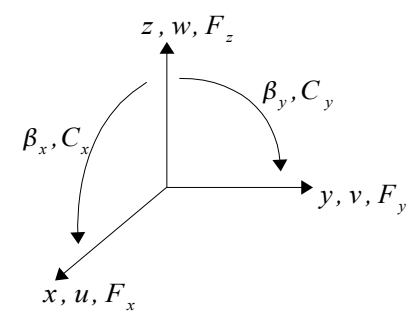

Figure 1. Conventions de signe pour les déplacements, rotations, forces et couples 
Si la surface moyenne non déformée est le plan $z=0$, le déplacement d'un point $P(x, y, z)$ est donné par le vecteur [6] :

$$
\left\{\begin{array}{c}
u(x, y)+z \beta_{x}(x, y) \\
v(x, y)+z \beta_{y}(x, y) \\
w(x, y)
\end{array}\right\}
$$

Par dérivation de ce champ de déplacements, on en déduit les déformations «planes » [7] et les déformations « de cisaillement transversal » [8] :

$$
\begin{aligned}
& \left\{\begin{array}{c}
\epsilon_{x} \\
\epsilon_{y} \\
2 \epsilon_{x y}
\end{array}\right\}=\left\{\begin{array}{c}
u_{, x} \\
v_{, y} \\
u_{, y}+v_{, x}
\end{array}\right\}+z\left\{\begin{array}{c}
\beta_{x, x} \\
\beta_{y, y} \\
\beta_{x, y}+\beta_{y, x}
\end{array}\right\} \\
& \left\{\begin{array}{l}
\gamma_{x} \\
\gamma_{y}
\end{array}\right\}=\left\{\begin{array}{l}
2 \epsilon_{x z} \\
2 \epsilon_{y z}
\end{array}\right\}=\left\{\begin{array}{l}
\beta_{x}+w_{, x} \\
\beta_{y}+w_{, y}
\end{array}\right\}
\end{aligned}
$$

La loi de comportement du matériau fournit ensuite les efforts de plaque correspondants : les efforts normaux [9], les moments [10] et les efforts tranchants [11]. Dans ce qui suit, on se limite aux matériaux homogènes, isotropes et élastiques linéaires. Cette hypothèse permet notamment de découpler les fonctionnements membranaire et de flexion/cisaillement transversal de la plaque. On obtient ainsi, avec les conventions indiquées sur la figure 2 :

$$
\begin{aligned}
& \left\{\begin{array}{l}
N_{x} \\
N_{y} \\
N_{x y}
\end{array}\right\}=\frac{E h}{\left(1-v^{2}\right)}\left(\begin{array}{ccc}
1 & v & 0 \\
v & 1 & 0 \\
0 & 0 & \frac{1-v}{2}
\end{array}\right)\left\{\begin{array}{c}
u_{, x} \\
v, y \\
u_{, y}+v_{, x}
\end{array}\right\} \\
& \left\{\begin{array}{c}
M_{x} \\
M_{y} \\
M_{x y}
\end{array}\right\}=\frac{E h^{3}}{12\left(1-v^{2}\right)}\left(\begin{array}{ccc}
1 & v & 0 \\
v & 1 & 0 \\
0 & 0 & \frac{1-v}{2}
\end{array}\right)\left\{\begin{array}{c}
\beta_{x, x} \\
\beta_{y, y} \\
\beta_{x, y}+\beta_{y, x}
\end{array}\right\} \\
& \left\{\begin{array}{c}
T_{x} \\
T_{y}
\end{array}\right\}=\frac{5}{6} \frac{E h}{2(1+v)}\left(\begin{array}{ll}
1 & 0 \\
0 & 1
\end{array}\right)\left\{\begin{array}{l}
\beta_{x}+w_{, x} \\
\beta_{y}+w_{, y}
\end{array}\right\}
\end{aligned}
$$

où le coefficient $\frac{5}{6}$ est une correction apportée au modèle de plaque de façon à ce que l'énergie interne de cisaillement transversal résultant de cette théorie de plaque corresponde à celle issue d'un véritable modèle élastique tridimensionnel (Batoz, 1990). 

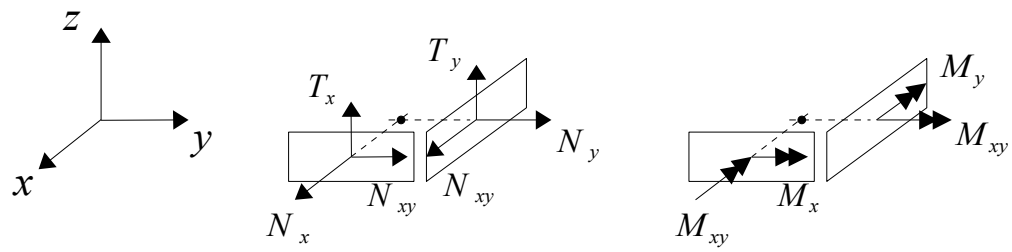

Figure 2. Conventions de signe pour les efforts de plaque

\subsubsection{Calcul des surfaces d'influence d'efforts par la méthode des éléments finis}

Dans la perspective de l'application au calcul des surfaces d'influence dans les ponts-dalle, on se restreint ici aux influences de la composante d'action $F_{z}$ (force verticale s'exerçant sur le tablier du pont) sur les efforts dans une plaque horizontale.

Dans un calcul de plaque par éléments finis, les nœuds portent en général comme degrés de liberté les déplacements de membrane $u^{i}, v^{i}$ et les déplacements de flexion $w^{i}, \beta_{x}^{i}, \beta_{y}^{i}$. Afin de ne pas alourdir l'exposé, on ne s'intéresse dans ce qui suit qu'au fonctionnement de flexion, en omettant volontairement le fonctionnement membranaire (on rappelle que compte-tenu de l'hypothèse d'homogénéité et d'isotropie effectuée sur le matériau, ces 2 fonctionnements sont découplés).

Comme il a été signalé en section 3.1.2 (expressions [4] et [5]), il est facile de déterminer l'influence d'une action sur une combinaison linéaire $\sum_{k \in K} c_{1 \mathrm{k}}(P) w^{k}+c_{2 \mathrm{k}}(P) \beta_{x}^{k}+c_{3 \mathrm{k}}(P) \beta_{y}^{k}$. Or, la méthode des éléments finis conduit précisément à approximer les efforts de plaque par des expressions de ce type.

Détaillons le principe d'obtention de telles expressions, en séparant les cas où le point d'étude coïncide ou non avec un nœud.

\section{Cas où le point d'étude ne coïncide pas avec un nœud}

L'approximation par éléments finis consiste à considérer des approximations nodales à l'intérieur de chacune des mailles (élément fini) du modèle : par exemple, pour une maille à $q$ nœuds et pour un point $P$ de cette maille, l'effort $E^{P}$ en ce point s'exprime sous la forme [12] :

$$
E^{P}=\sum_{k=1}^{q} c_{1 \mathrm{k}}(P) w^{k}+c_{2 \mathrm{k}}(P) \beta_{x}^{k}+c_{3 \mathrm{k}}(P) \beta_{y}^{k}
$$

où les coefficients $c_{1 \mathrm{k}}(P), c_{2 \mathrm{k}}(P), c_{3 \mathrm{k}}(P)$ dépendent de la maille considérée et de la position du point $P$ dans la maille.

La surface d'influence de $E^{P}$ s'obtient donc en appliquant l'action duale [13] :

$$
\sum_{k=1}^{q} c_{1 \mathrm{k}}(P) F_{z}^{k}+c_{2 \mathrm{k}}(P) C_{x}^{k}+c_{3 \mathrm{k}}(P) C_{y}^{k}
$$


La démarche d'obtention des coefficients $c_{i k}(P)$ est simple mais parfois lourde: on part de l'approximation nodale des déplacements, pour obtenir successivement les approximations nodales des déformations (par dérivations), des contraintes (par loi de comportement) puis enfin des efforts (par intégration sur l'épaisseur de la plaque).

L'annexe 1 donne les expressions de tels coefficients dans le cas de l'élément de plaque Discrete Shear Quadrangle et pour tous les efforts de flexion (moments et efforts tranchants), en utilisant une présentation matricielle.

\section{Cas où le point d'étude est un nœud}

Notons $i$ ce nœud et $E^{i}$ l'effort considéré. Si on choisit une maille $m$ contenant $i$, on obtient, en suivant la démarche précédente, une expression du type [14] :

$$
E^{i}=\sum_{k=1}^{q} c_{1 \mathrm{k}} w^{k}+c_{2 \mathrm{k}} \beta_{x}^{k}+c_{3 \mathrm{k}} \beta_{y}^{k}
$$

Cette expression dépendant du choix de la maille $m$, on la note donc plutôt $E^{i, m}$.

Suivant le choix effectué pour la maille $m$, on obtient des valeurs $E^{i, m}$ différentes. Ceci est dû au fait que le champ d'effort obtenu par juxtaposition des approximations nodales dans les différentes mailles du modèle n'est en général pas continu à la frontière entre mailles. C'est pourquoi on conseille de procéder à un lissage : considérer la moyenne arithmétique $E^{i}$ des valeurs $E^{i, m}$ obtenues pour toutes les mailles $m$ contenant $i$, constitue une approximation suffisante lorsque le maillage est régulier (dans le cas d'éléments de tailles différentes, on peut considérer une moyenne pondérée par les tailles des éléments).

Ce lissage conduit donc encore en définitive à une relation linéaire du type [15] :

$$
E^{i}=\sum_{k=1}^{\lambda} c_{1 \mathrm{k}} w^{k}+c_{2 \mathrm{k}} \beta_{x}^{k}+c_{3 \mathrm{k}} \beta_{y}^{k}
$$

mais impliquant un nombre plus grand de nœuds : $\lambda>q$

\section{Application de la méthode à un pont-dalle}

\subsection{Cas d'étude : surfaces d'influence de "ponts-dalle"}

On s'intéresse à l'étude des ponts-dalle, qui constituent un type d'ouvrage très fréquemment utilisé, notamment pour les passages supérieurs ou inférieurs autoroutiers. Il s'agit d'une structure simple dont le tablier, constitué d'une dalle en béton, repose sur ses appuis (les piles et les culées) par l'intermédiaire de blocs parallélépipédiques en élastomère fretté appelés appareils d'appui. L'ensemble des appareils d'appui d'un même appui sont en général alignés et constituent une ligne 
d'appui. L'angle formé par la direction longitudinale de la dalle et la direction d'une ligne d'appui est appelé le biais géométrique de l'appui. Traditionnellement mesuré en grades (100 grades $=90$ degrés), le biais constitue un paramètre important du fonctionnement mécanique des ponts-dalle. Ce biais peut être variable d'une ligne d'appui à l'autre. Dans le cas contraire, le biais est dit constant.

Signalons que l'étude des ponts-dalle a pendant longtemps consisté à réaliser un calcul longitudinal («en poutre »), doublé d'un calcul de répartition transversale fondé sur la méthode des coefficients de répartition de Guyon-Massonnet-Barès (1966). L'emploi de cette méthode simplifiée permet en particulier de se contenter de produire et d'exploiter des lignes d'influence plutôt que des surfaces. Cependant, cette méthode présente des limites d'utilisation importantes : en particulier, le calcul de répartition transversale suppose que le pont est droit (biais de 100 grades) ou en tout cas peu biais (biais couramment compris entre 100 et 75 grades). En revanche, le calcul aux éléments finis permet de traiter indifféremment n'importe quel cas de biais.

Deux ponts-dalle présentant chacun un biais constant ont été étudiés :

- l'un de 100 grades, le pont étant alors dit droit (figure 3),

- l'autre de 50 grades, le pont étant alors dit de biais modéré (figure 4).

En un certain nombre de points d'étude et pour chaque effort de dalle (moments, efforts tranchants), la modélisation numérique a consisté :

- à déterminer puis mettre en œuvre le chargement fictif générant la surface correspondante,

- à mettre en œuvre une procédure de validation de la surface ainsi générée, par comparaison avec des calculs directs.

On considère des ponts à 3 travées $(14 \mathrm{~m}-20 \mathrm{~m}-14 \mathrm{~m})$ munis d'abouts (surlongueurs aux deux extrémités du pont) de 0,60 m (resp. 1,20 m) dans le cas du pont droit (resp. de biais modéré), et présentant une largeur de $10 \mathrm{~m}$ correspondant à un profil en travers classique : 2 voies de 3,50 m et 2 trottoirs de 1,50 m. La dalle présente un élancement de $\frac{1}{28}$, soit une épaisseur de $71 \mathrm{~cm}$.

La dalle est en béton, avec un module d'Young de $11000000 \mathrm{kN} / \mathrm{m}^{2}$ et un coefficient de Poisson de 0,2 .

On considère 5 points d'étude (A à E). Le point $\mathrm{A}$ est disposé au milieu de la travée centrale, tandis que les 4 autres points sont disposés alignés le long d'un bord longitudinal de la dalle. Le point $\mathrm{C}$ correspond à un appareil d'appui tandis que les points $\mathrm{D}$ et $\mathrm{E}$ sont placés à sa proximité. Les figures 3 et 4 illustrent les différentes positions des points d'étude. 


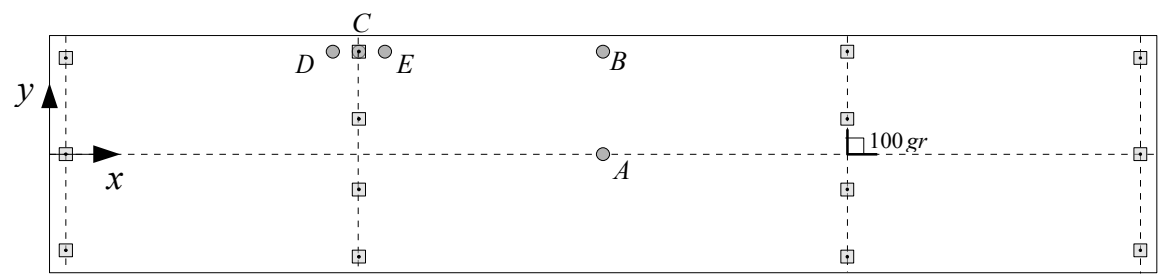

Figure 3. Vue en plan du pont-dalle droit, avec ses appareils d'appui et ses points d'étude $A$ à $E$

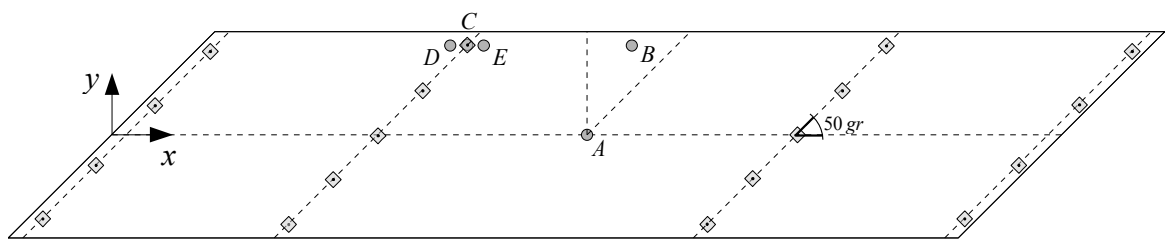

Figure 4. Vue en plan du pont-dalle de biais modéré, avec ses appareils d'appui et ses points d'étude $A$ à $E$

Les modèles aux éléments finis ont été réalisés à l'aide du logiciel Code_Aster. Il s'agit d'un code général pour l'étude du comportement mécanique des structures, développé par EDF R\&D. Il est diffusé sous licence GNU GPL sous l'appellation Code_Aster Libre (http://www.code-aster.org).

En particulier, Code_Aster Libre dispose de la formulation d'élément de plaque « épaisse » Discrete Shear applicable à des mailles support triangle ou quadrangle. Les détails de la formulation sont fournis dans la documentation de référence du code (Massin, 2001) dont l'annexe 1 reprend une partie des notations.

\subsection{Détails de mise en oeuvre}

\subsubsection{Maillage}

Compte tenu de la géométrie fort simple du domaine à mailler, les maillages des 2 ponts-dalle sont constitués : 
- soit de rectangles, dans le cas du pont droit,

- soit de parallélogrammes, dans le cas du pont de biais modéré.

Par ailleurs, la finesse du maillage est telle que la taille d'un côté d'une maille correspond environ à la demi-épaisseur de dalle.

\subsubsection{Prise en compte des appareils d'appui}

Les appareils d'appui sont introduits dans le modèle éléments finis de façon simplifiée, sous la forme d'appuis ponctuels rigides : les déplacements verticaux des nœuds correspondants sont bloqués à 0 .

\subsubsection{Génération des surfaces d'influence}

On considère le cas où le point d'étude $P_{e}$ correspond à un nœud du maillage. Il s'agit du cas le plus délicat dans la mesure où il nécessite une étape de lissage, comme on l'a signalé en section 3.2.2 (relation [15]). Dans la configuration présente, la dalle étant maillée régulièrement en rectangles ou en parallélogrammes (selon son biais), 4 mailles contiennent $P_{e}$. Par suite, 4 chargements peuvent être considérés pour générer la surface cherchée (1 chargement par choix de maille). Néanmoins, dans un souci de lissage, le chargement appliqué est constitué de la moyenne arithmétique de ces 4 chargements, ce qui conduit à charger les 9 nœuds « centrés » sur $P_{e}$ (figure 5a).

Dans le cas plus simple d'un point d'étude $P_{e}$ à l'intérieur d'une maille, on utiliserait la relation bilinéaire issue directement de l'approximation nodale associée à la maille, et par conséquent, seuls 4 nœuds seraient chargés (figure 5b).

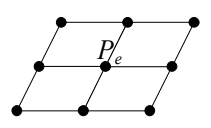

Figure 5a. Nouds chargés si $P_{e}$ est un noud du maillage (9 nœuds)

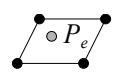

Figure 5b. Nouds chargés si $P_{e}$ est à l'intérieur d'une maille (4 nœuds) 
508 Revue européenne de mécanique numérique. Volume 17 - n 4/2008
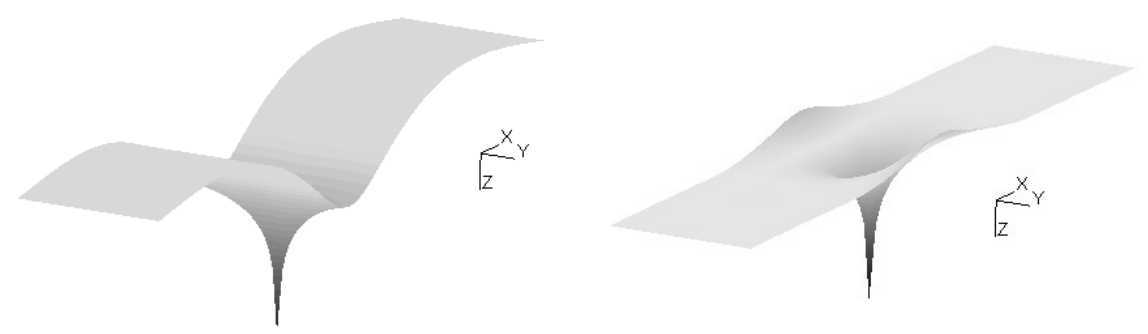

Figure 6a. Surface d'influence de $M_{x}$ au point $A$

Figure 6b. Surface d'influence de $M_{y}$ au point $A$

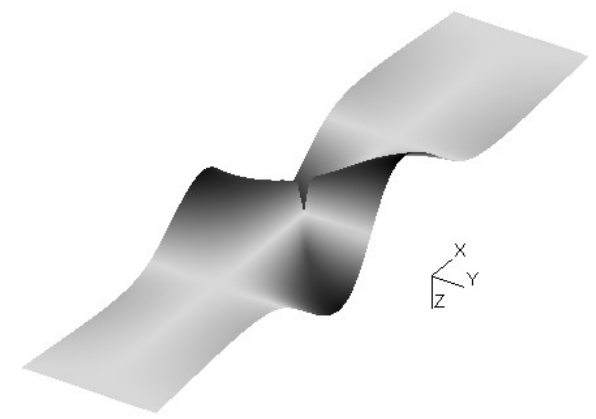

Figure 6c. Surface d'influence de $M_{x y}$ au point $A$

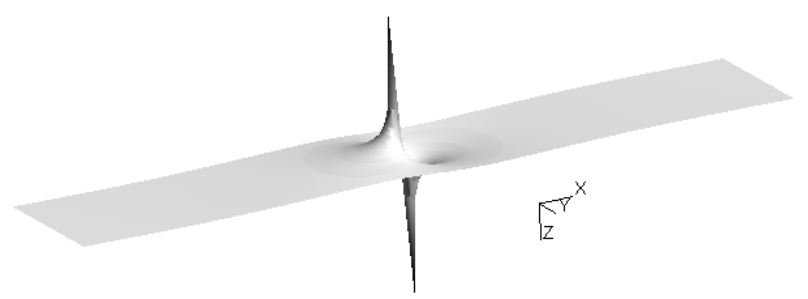

Figure 6d. Surface d'influence de $T_{x}$ au point $A$ 


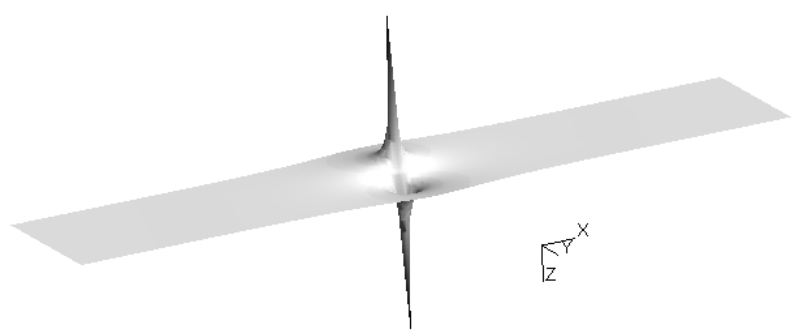

Figure 6e. Surface d'influence de $T_{y}$ au point $A$

\subsection{Résultats}

4.3.1. Allures de quelques surfaces d'influence dans le cas du pont droit, lorsque l'action unitaire est une force verticale unitaire dirigée vers les $z$ positifs

Les surfaces d'influence des moments $M_{x}$ et $M_{y}$ présentent des «pics» de valeurs au droit du point d'étude. Ces pics rendent compte de la singularité (valeur infinie) des champs de $M_{x}$ et $M_{y}$ en $P_{e}$ lorsqu'on applique une force ponctuelle en $P_{e}$.

Les surfaces d'influence des efforts tranchants présentent une succession de deux pics inversés au droit du point d'étude. Cette inversion rend compte d'une discontinuité de la surface recherchée, celle-ci étant liée à la singularité en $P_{e}$ des champs de $M_{x}$ et $M_{y}$ comme le montrent les relations d'équilibre interne (rappelées en annexe 1) reliant, par dérivation, les efforts tranchants aux moments.

\subsubsection{Vérification de la propriété de réciprocité}

Si l'on note :

- $E\left(P_{e}\right)$ la valeur de l'effort considéré au point d'étude $P_{e}$, sous une densité surfacique de force verticale $f$ s'appliquant sur une zone $\omega$ du tablier,

- $E\left(P_{e}, P\right)$ la valeur courante au point $P$ de la surface d'influence de l'effort $E$ en $P_{e}$,

alors on dit que la propriété de réciprocité de la surface est vérifiée si on a l'égalité [16] :

$$
\iint_{\omega} f(P) E\left(P_{e}, P\right) d \omega(P)=E\left(P_{e}\right)
$$

Le principe de validation des surfaces d'influence consiste alors à tester, pour certains cas de charge, c'est-à-dire pour certaines valeurs de $\omega$ et $f$, si la propriété de réciprocité des surfaces est vérifiée. 
Les cas de charges considérés prennent en compte une densité $f$ constante unitaire et appliquée sur une zone $\omega$ correspondant :

- soit à toute la surface de la dalle,

- soit à une zone carrée dite d'impact (de côté 1,20 m) disposée de façon à produire l'effet le plus défavorable en $P_{e}$.

Ceci permet ainsi de tester la qualité de la surface générée, d'une part, globalement, et d'autre part, localement au voisinage du point d'étude.

Pour le cas de charge impact, la position de l'impact produisant l'effet le plus défavorable en $P_{e}$ dépend de l'effet considéré et est suggérée par l'allure de la surface d'influence correspondante au voisinage de $P_{e}$. On s'efforce de disposer l'impact sur une zone de la surface présentant de fortes valeurs. On indique sur les figures $7 \mathrm{a}$ à $7 \mathrm{~d}$ les positions considérées dans chacun des cas.

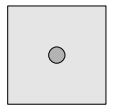

Figure 7a. Position de l'impact utilisé pour $M_{x}$ et $M_{y}$. L'impact est centré sur le point d'étude

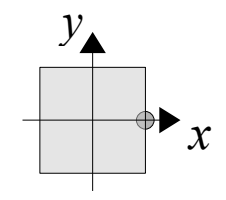

Figure 7c. Position de l'impact utilisé pour $T_{x}$. L'impact reste dans le $1 / 2$ plan $x \leqslant x\left(P_{e}\right)$
Figure 7b. Position de l'impact utilisé pour $M_{x y}$. L'impact est excentré par rapport au point d'étude

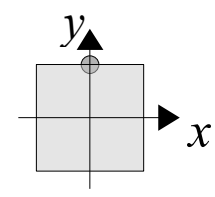

Figure 7d. Position de l'impact utilisé pour $T_{y}$. L'impact reste dans le $1 / 2$ plan $y \leqslant y\left(P_{e}\right)$

Pour chaque cas de charge, on observe la précision avec laquelle les surfaces d'influence vérifient la propriété de réciprocité, en comparant les valeurs issues du calcul direct sous le cas de charge, i.e. $E\left(P_{e}\right)$, avec les valeurs issues de l'intégration de la surface, i.e. $\iint_{\omega} f E\left(P_{e}, P\right) d P$. Dans chaque situation, il s'avère que l'écart relatif reste très inférieur à $1 \%$.

Les tableaux $1 \mathrm{a}$ et $1 \mathrm{~b}$ présentent les résultats pour le point d'étude A. Les résultats pour les autres points d'étude figurent en Annexe 2. 


\begin{tabular}{|c|c|c|c|c|}
\hline \multirow{3}{*}{$\begin{array}{c}\text { Point d'étude } \\
\text { A }\end{array}$} & \multirow{2}{*}{\multicolumn{2}{|c|}{$\begin{array}{l}\text { Pont droit } \\
x=24,45 \quad y=0\end{array}$}} & \multicolumn{2}{|c|}{ Pont de biais modéré } \\
\hline & & & $x=25,0$ & $y=0$ \\
\hline & $\begin{array}{l}\text { Intégration } \\
\text { influences }\end{array}$ & $\begin{array}{l}\text { Calcul } \\
\text { direct }\end{array}$ & $\begin{array}{l}\text { Intégration } \\
\text { influences }\end{array}$ & $\begin{array}{l}\text { Calcul } \\
\text { direct }\end{array}$ \\
\hline $\begin{array}{c}M_{x} \\
(\mathrm{kN} \cdot \mathrm{m} / \mathrm{m})\end{array}$ & 19,1638 & 19,1638 & 13,0750 & 13,0750 \\
\hline $\begin{array}{c}M_{y} \\
(\mathrm{kN} \cdot \mathrm{m} / \mathrm{m})\end{array}$ & 2,0862 & 2,0862 & 3,3258 & 3,3258 \\
\hline $\begin{array}{c}M_{x y} \\
(\mathrm{kN} \cdot \mathrm{m} / \mathrm{m})\end{array}$ & 0,0000 & 0,0000 & $-5,7571$ & $-5,7571$ \\
\hline $\begin{array}{c}T_{x} \\
(\mathrm{kN} / \mathrm{m})\end{array}$ & 0,1367 & 0,1367 & 0,1164 & 0,1164 \\
\hline $\begin{array}{c}T_{y} \\
(\mathrm{kN} / \mathrm{m})\end{array}$ & 0,0000 & 0,0000 & $-0,0501$ & $-0,0501$ \\
\hline
\end{tabular}

Tableau 1a. Comparaison du calcul direct et de l'intégration des influences pour le point d'étude $A$ : chargement de toute la surface

\begin{tabular}{|c|c|c|c|c|}
\hline \multirow{3}{*}{$\begin{array}{c}\text { Point d'étude } \\
\text { A }\end{array}$} & \multirow{2}{*}{\multicolumn{2}{|c|}{$\begin{array}{l}\text { Pont droit } \\
x=24,45 \quad y=0\end{array}$}} & \multicolumn{2}{|c|}{ Pont de biais modéré } \\
\hline & & & $x=25,0$ & $y=0$ \\
\hline & $\begin{array}{l}\text { Intégration } \\
\text { influences }\end{array}$ & $\begin{array}{l}\text { Calcul } \\
\text { direct }\end{array}$ & $\begin{array}{l}\text { Intégration } \\
\text { influences }\end{array}$ & $\begin{array}{l}\text { Calcul } \\
\text { direct }\end{array}$ \\
\hline $\begin{array}{c}M_{x} \\
(\mathrm{kN} \cdot \mathrm{m} / \mathrm{m})\end{array}$ & 0,5313 & 0,5313 & 0,4896 & 0,4897 \\
\hline $\begin{array}{c}M_{y} \\
(\mathrm{kN} \cdot \mathrm{m} / \mathrm{m})\end{array}$ & 0,2900 & 0,2900 & 0,3465 & 0,3465 \\
\hline $\begin{array}{c}M_{x y} \\
(\mathrm{kN} \cdot \mathrm{m} / \mathrm{m})\end{array}$ & $-0,0629$ & $-0,0629$ & $-0,1447$ & $-0,1447$ \\
\hline $\begin{array}{c}T_{x} \\
(\mathrm{kN} / \mathrm{m})\end{array}$ & $-0,2625$ & $-0,2625$ & $-0,2215$ & $-0,2215$ \\
\hline $\begin{array}{c}T_{y} \\
(\mathrm{kN} / \mathrm{m})\end{array}$ & $-0,2749$ & $-0,2749$ & $-0,2541$ & $-0,2541$ \\
\hline
\end{tabular}

Tableau 1b. Comparaison du calcul direct et de l'intégration des influences pour le point d'étude A : chargement d'une zone d'impact 


\subsubsection{Exemple d'utilisation des surfaces d'influence générées}

Considérons à titre d'illustration le cas d'une charge mobile circulant sur le pont droit présenté en figure 3. Cette charge est constituée d'un essieu comportant 2 roues de $200 \mathrm{kN}$, de dimensions longitudinale $0,35 \mathrm{~m}$ et transversale $0,60 \mathrm{~m}$ espacées entre axes de $2 \mathrm{~m}$. Cet essieu peut se déplacer sur la chaussée, le long de files longitudinales telles que les 2 roues de l'essieu soient toujours entièrement positionnées sur la chaussée (pas de roue sur les trottoirs). Au point d'étude A (centre de la travée centrale), on recherche la valeur absolue maximale du moment de flexion transversale $M_{y}$ due à la circulation de cette charge, ainsi que la position concomitante de la charge.

Le pont étant représenté dans le modèle éléments finis par le plan moyen de la dalle, il convient de prendre en compte une diffusion des charges depuis leur zone d'application (partie supérieure de la chaussée), jusqu'à la surface moyenne de la dalle : en considérant que la chaussée présente une épaisseur de $4,5 \mathrm{~cm}$, la dalle une épaisseur de $71 \mathrm{~cm}$, et que la diffusion s'effectue suivant un angle de 45 degrés, on obtient, après diffusion, 2 impacts de dimensions longitudinale $1,15 \mathrm{~m}$ et transversale 1,40 m. La figure 8a illustre ce principe de diffusion de la charge en coupe longitudinale. Sur chaque impact s'exerce donc une pression de 124,2 kPa $\left(\simeq \frac{200}{1,15 \cdot 1,40}\right)$.

Pour chaque position possible de la charge mobile sur la chaussée, le moment $M_{y}$ au point A s'obtient par l'intégrale [17] de la surface d'influence de $M_{y}$ au point $\mathrm{A}$ (figure $6 \mathrm{~b}$ ) sur les 2 impacts associés à la charge :

$$
\iint_{\text {impacts }} 124,2 \cdot M_{y}(A, P) d \omega(P)
$$

La figure $8 \mathrm{~b}$ montre le positionnement limite de la charge côté bord gauche de la chaussée.

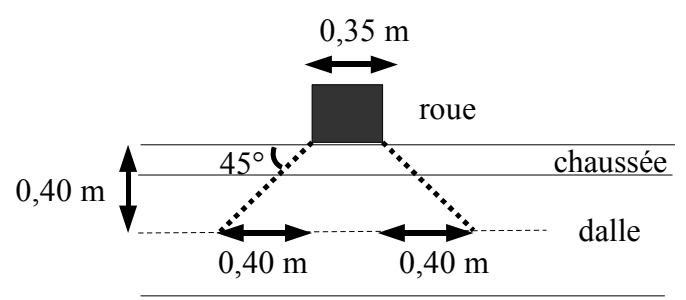

Figure 8a. Diffusion de la charge en coupe longitudinale 


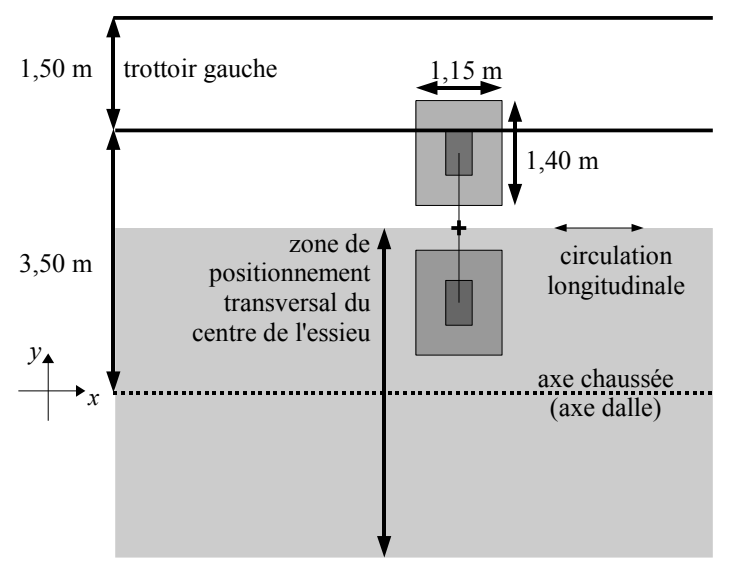

Figure 8b. Position limite, côté bord gauche de la chaussée, de la charge mobile considérée

L'examen visuel de la surface d'influence (figure 6b) permet de cerner la position recherchée :

- longitudinalement, la charge doit être disposée à la même abscisse que le point A (soit $x=24,45$ ),

- transversalement, on pressent que le centre de l'essieu doit être disposé de façon excentrée par rapport à l'ordonnée du point $\mathrm{A}$, de façon à ce qu'une des 2 roues se situe au voisinage du pic de la surface (soit $y \simeq \pm 1,00$ ); il y a donc 2 positions transversales possibles, symétriques par rapport à l'axe du pont.

L'exploitation quantitative de la surface d'influence (intégrations) permet de préciser la position transversale. Le tableau 2 fournit le moment maximal obtenu ainsi que les 2 positions concomitantes de la charge.

\begin{tabular}{|c|c|c|}
\hline \multirow{2}{*}{$\begin{array}{c}\text { Point d'étude } \\
\text { A }\end{array}$} & \multicolumn{2}{|c|}{ Pont droit } \\
\cline { 2 - 3 } & $\begin{array}{c}\text { Valeur absolue maximale } \\
\text { du moment } M_{y} \\
(\mathrm{kN} . \mathrm{m} / \mathrm{m})\end{array}$ & $\begin{array}{c}\text { Positions concomitantes } \\
\text { de la charge mobile }\end{array}$ \\
$\begin{array}{c}x=0 \\
y=0\end{array}$ & 52,999 & $x=24,45$ \\
& & $y= \pm 0,715$ \\
\hline
\end{tabular}

Tableau 2. Valeur absolue maximale du moment $M_{y}$ au point d'étude $A d u$ pont droit sous l'action d'une charge mobile se déplaçant sur la chaussée 
On constate donc un léger décalage par rapport aux positions transversales pressenties. La figure 9 fournit une représentation graphique des moments $-M_{y}$ au point $\mathrm{A}$, pour les différentes positions de la charge mobile. La représentation des valeurs de $-M_{y}$ permet une comparaison de forme avec la surface d'influence de la figure 6b. Il s'agit ici d'une surface d'influence "généralisée », au sens où la charge qui se déplace n'est pas ponctuelle unitaire mais «réelle» (surfacique, non unitaire, dirigée vers les $z$ négatifs).

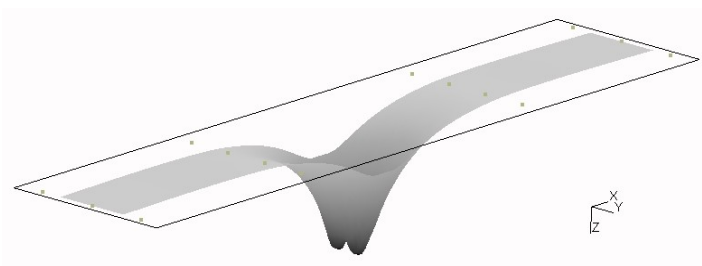

Figure 9. Surface d'influence généralisée de $-M_{y}$ au point A pour la charge mobile considérée

Bien entendu, l'exemple traité ici reste très simple. Dans la pratique, l'application des règlements de charges (du type Eurocode 1) conduit à déplacer simultanément différents types de charges mobiles. Compte-tenu de l'ampleur de la tâche, la recherche systématique des positions conduisant aux effets extrêmaux, nécessite l'emploi de logiciels spécifiques intégrant le règlement de charges à appliquer.

\section{Conclusion}

Cet article illustre l'utilisation de la formulation de l'élément fini de plaque épaisse «Discrete Shear Quadrangle» pour la génération numérique des surfaces d'influence des efforts de plaque.

La notion d'influence est une notion essentielle au calcul des structures soumises à des charges mobiles telles que les ponts routiers.

Le calcul numérique des fonctions d'influence peut être réalisé par application du théorème de réciprocité de Maxwell-Betti, quelle que soit la méthode matricielle de calcul structural envisagée. Très répandu dans le cas des ponts élancés relevant d'une modélisation filaire (lignes d'influence), ce type de calcul est également à la base du calcul des ponts courants relevant plus d'une modélisation surfacique (surfaces d'influence). 
Les tests mis en œuvre pour valider les surfaces générées dans le cas de 2 pontsdalle ont permis de vérifier, avec une très bonne précision, les propriétés recherchées de réciprocité.

Par rapport à l'utilisation traditionnelle de catalogues d'abaques, l'utilisation d'un modèle de calcul numérique présente l'avantage de pouvoir générer les surfaces d'influence directement adaptées à la géométrie de la structure étudiée, alors qu'un catalogue d'abaques ne propose qu'un choix limité de configurations géométriques.

Néanmoins, sans outils complémentaires, l'exploitation des surfaces générées reste fastidieuse. La tendance actuelle consiste à coupler le logiciel de calcul de structure avec un logiciel d'exploitation de surfaces d'influence, intégrant les contraintes propres à un ou plusieurs règlements de charges routières, de façon à pouvoir réaliser automatiquement et systématiquement la recherche des distributions de trafic conduisant aux effets structuraux extrêmes.

\section{Bibliographie}

Barès R., Massonnet C., Le calcul des grillages de poutres et dalles orthotropes selon la méthode Guyon-Massonnet-Barès, Paris, Dunod, 1966.

Batoz J.L., Dhatt G., Modélisation des structures par éléments finis (volume 2 : Poutres et plaques), Paris, Hermès, 1990.

Brisard S., « Abaques pour la flexion locale de la dalle d'un bipoutre à entretoises », Bulletin $d u$ Centre des Techniques d'Ouvrages d'Art, $\mathrm{n}^{\circ}$ 54, 2007, p. 10-21.

Jenssen V. P., «Analyses of skew slabs », University of Illinois Bulletin, vol. XXXIX, n 3, 1941, p. 79-81.

Le Faucheur D., «Flexion locale dans les hourdis de ponts ", Bulletin du Centre des Techniques d'Ouvrages d'Art, n 49, 2005, p. 12-17.

Massin P., «Eléments de plaque DKT, DST, DKQ, DSQ et Q4g », Manuel de référence du Code_Aster, R3.07.03-A, 2001.

Orakdögen E., Girgin K., « Direct determination of influence lines and surfaces by F.E.M. », Structural Engineering \& Mechanics, vol. 20, n 3, 2005, p. 279-292.

Rüsh H., Hergenröder A., Einflussfelder der momente, schiefwinkliger pattern, TH München, 1964.

Zienkiewitcz O.C., La méthode des éléments finis appliquée à l'art de l'ingénieur, Paris, McGraw-Hill, 1973. 


\section{Annexe 1 : matrices $I M$ et $I T$ de l'élément DSQ}

La formulation « Discrete Shear » est décrite par Batoz (1990) et reprise en détail dans la documentation du Code_Aster (Massin, 2001). On considère ici des quadrangles à 4 nœuds.

\subsection{Approximation de la géométrie}

Le passage de l'élément de référence (pavé $[-1,1]^{2}$ ) à l'élément réel s'effectue au moyen des formules [18] et [19] de transformation géométrique :

$$
\begin{aligned}
& x(\xi, \eta)=\sum_{i=1}^{4} N_{i}(\xi, \eta) x_{i} \\
& y(\xi, \eta)=\sum_{i=1}^{4} N_{i}(\xi, \eta) y_{i}
\end{aligned}
$$

où :

- $x_{i}$ et $y_{i}$ sont les coordonnées des nœuds de l'élément réel,

- $(\xi, \eta)$ représentent les coordonnées dans l'élément de référence,

- les fonctions de forme sont données par les expressions [20]-[23] :

$$
\begin{aligned}
& N_{1}(\xi, \eta)=\frac{1}{4}(1-\xi)(1-\eta) \\
& N_{2}(\xi, \eta)=\frac{1}{4}(1+\xi)(1-\eta) \\
& N_{3}(\xi, \eta)=\frac{1}{4}(1+\xi)(1+\eta) \\
& N_{4}(\xi, \eta)=\frac{1}{4}(1-\xi)(1+\eta)
\end{aligned}
$$

\subsection{Approximation des déplacements}

La discrétisation [24] des déplacements de la surface moyenne (les flèches, puisqu'on se restreint aux plaques en flexion avec cisaillement transversal) est bilinéaire :

$$
w(\xi, \eta)=\sum_{i=1}^{4} N_{i}(\xi, \eta) w^{i}
$$


où $w^{i}$ sont les flèches aux nœuds de l'élément, tandis que les discrétisations [25] - [26] des rotations de la normale à la surface moyenne sont quadratiques et font intervenir des paramètres $\alpha_{k}$ qui s'élimineront au cours de la résolution :

$$
\begin{aligned}
& \beta_{x}(\xi, \eta)=\sum_{i=1}^{4} N_{i}(\xi, \eta) \beta_{x}^{i}+\sum_{k=1}^{4} P_{k}(\xi, \eta) c_{k} \alpha_{k} \\
& \beta_{y}(\xi, \eta)=\sum_{i=1}^{4} N_{i}(\xi, \eta) \beta_{y}^{i}+\sum_{k=1}^{4} P_{k}(\xi, \eta) s_{k} \alpha_{k}
\end{aligned}
$$

où :

- $\beta_{x}^{i}$ et $\beta_{y}^{i}$ sont les rotations aux nœuds de l'élément,

- $\quad k$ indice les côtés de l'élément : $\quad k=1 \quad$ (resp. 2, 3, 4) relie le nœud 1 (resp. 2, 3, 4) au nœud 2 (resp. 3, 4, 1),

$-c_{k}$ et $s_{k}$ sont les cosinus et sinus directeurs des côtés de l'élément,

- les fonctions de formes supplémentaires sont données par les expressions [27] - [30] :

$$
\begin{aligned}
& P_{1}(\xi, \eta)=\frac{1}{2}\left(1-\xi^{2}\right)(1-\eta) \\
& P_{2}(\xi, \eta)=\frac{1}{2}\left(1-\eta^{2}\right)(1+\xi) \\
& P_{3}(\xi, \eta)=\frac{1}{2}\left(1-\xi^{2}\right)(1+\eta) \\
& P_{4}(\xi, \eta)=\frac{1}{2}\left(1-\eta^{2}\right)(1-\xi)
\end{aligned}
$$

\subsection{Approximation des efforts (matrices $I M$ et $I T$ )}

L'approximation nodale recherchée des moments [31] et des efforts tranchants [32] s'exprime matriciellement : 


$$
\begin{gathered}
\left\{\begin{array}{l}
M_{x} \\
M_{y} \\
M_{x y}
\end{array}\right\}=I M\left\{\begin{array}{c}
w^{1} \\
\beta_{x}^{1} \\
\beta_{y}^{1} \\
\vdots \\
w^{4} \\
\beta_{x}^{4} \\
\beta_{y}^{4}
\end{array}\right) \\
\left\{\begin{array}{l}
T_{x} \\
T_{y}
\end{array}\right\}=I T\left\{\begin{array}{c}
w^{1} \\
\beta_{x}^{1} \\
\beta_{y}^{1} \\
\vdots \\
w^{4} \\
\beta_{x}^{4} \\
\beta_{y}^{4}
\end{array}\right)
\end{gathered}
$$

où la matrice $I M$ (resp. IT ) de dimension 3x12 (resp. 2x12 ) dépend de la position du point $P$ dans la maille.

On déduit de ces expressions matricielles, les combinaisons linéaires exprimant chacun des efforts : par exemple, $M_{x}^{P}=\sum_{k=1}^{4} c_{1 \mathrm{k}} w^{k}+c_{2 \mathrm{k}} \beta_{x}^{k}+c_{3 \mathrm{k}} \beta_{y}^{k}$, où les coefficients $c_{1 \mathrm{k}}, c_{2 \mathrm{k}}, c_{3 \mathrm{k}}$ s'obtiennent à l'aide de la première ligne de $I M$.

Pour obtenir IM et $I T$, on procède de la façon suivante :

- pour la matrice $I M$, la démarche est «naturelle » au sens où l'on suit la progression classique (déplacements/déformations/efforts) : ainsi, la discrétisation des moments résulte directement de celle des courbures, c'est-à-dire des quantités [33] :

$$
\left\{\begin{array}{c}
\kappa_{x}=\beta_{x, x} \\
\kappa_{y}=\beta_{y, y} \\
\kappa_{x y}=\beta_{x, y}+\beta_{y, x}
\end{array}\right.
$$

- par contre, pour la matrice IT, la démarche est différente au sens où la discrétisation des efforts tranchants n'est pas déduite directement de celle des déformations de cisaillement transversale. Elle résulte en fait des relations [34]-[35] d'équilibre interne reliant les efforts tranchants aux moments :

$$
\begin{aligned}
& T_{x}=M_{x, x}+M_{x y, y} \\
& T_{y}=M_{y, y}+M_{x y, x}
\end{aligned}
$$

En définitive, on obtient IM et IT sous les formes générales [36]-[37] : 


$$
\begin{aligned}
& I M=H_{f} D\left(B_{f \beta}+B_{f \alpha} A_{\beta}\right) \\
& I T=B_{c \beta}+B_{c \alpha} A_{\beta}
\end{aligned}
$$

constituées chacune d'une somme de 2 termes :

- l'un associé aux déplacements de flexion $\left\langle w^{1}, \beta_{x}^{1}, \beta_{y}^{1}, \ldots, w^{4}, \beta_{x}^{4}, \beta_{y}^{4}\right\rangle$ (terme en $B_{f \beta}$ ou en $B_{c \beta}$ ),

- l'autre associé aux paramètres $\left\langle\alpha_{1}, \alpha_{2}, \alpha_{3}, \alpha_{4}\right\rangle$ (terme en $B_{f \alpha}$ ou en $\left.B_{c \alpha}\right)$.

Avant de détailler les différentes étapes du calcul de ces matrices, on rappelle que le passage de l'élément de référence à l'élément réel s'effectue au moyen d'une transformation géométrique de matrice jacobienne $\left(\begin{array}{ll}x_{, \xi} & y_{, \xi} \\ x_{, \eta} & y_{, \eta}\end{array}\right)$ dont l'inverse est notée dans ce qui suit $\left(\begin{array}{ll}j_{11} & j_{12} \\ j_{21} & j_{22}\end{array}\right)$.

\section{Etape 1 : matrice $H_{f} D$}

$H_{f} D$ est la matrice de rigidité de flexion, donnée par les expressions [38] - [39] :

$$
\begin{aligned}
& H_{f}=\frac{E h^{3}}{12\left(1-v^{2}\right)} \\
& D=\left(\begin{array}{ccc}
1 & v & 0 \\
v & 1 & 0 \\
0 & 0 & \frac{1-v}{2}
\end{array}\right)
\end{aligned}
$$

Etape 2 : matrice $B_{f \beta}$

La matrice $B_{f \beta}(3 \times 12)$ relie les courbures aux déplacements de flexion.

$$
B_{f \beta} \text { est définie par blocs : } B_{f \beta}=\left[\begin{array}{lllll}
{\left[B_{f \beta 1}\right]} & {\left[B_{f \beta 2}\right]} & {\left[B_{f \beta 3}\right]} & {\left[B_{f \beta 4}\right.}
\end{array}\right]
$$

avec des blocs de la forme [40] :

$$
B_{f \beta i}=\left(\begin{array}{ccc}
0 & j_{11} N_{i, \xi}+j_{12} N_{i, \eta} & 0 \\
0 & 0 & j_{21} N_{i, \xi}+j_{22} N_{i, \eta} \\
0 & j_{21} N_{i, \xi}+j_{22} N_{i, \eta} & j_{11} N_{i, \xi}+j_{12} N_{i, \eta}
\end{array}\right)
$$

Etape 3 : matrice $B_{f \alpha}$

La matrice $B_{f \alpha}(3 \times 4)$ relie les courbures aux paramètres $\alpha_{k}$. 
$B_{f \alpha} \quad$ est définie par blocs : $B_{f \alpha}=\left[\begin{array}{lllll}{\left[B_{f \alpha 1}\right]} & {\left[B_{f \alpha 2}\right]} & {\left[B_{f \alpha 3}\right]} & {\left[B_{f \alpha 4}\right.}\end{array}\right]$

avec des blocs de la forme [41] :

$$
B_{f \alpha i}=\left(\begin{array}{c}
j_{11} c_{i} P_{i, \xi}+j_{12} c_{i} P_{i, \eta} \\
j_{21} s_{i} P_{i, \xi}+j_{22} s_{i} P_{i, \eta} \\
j_{21} c_{i} P_{i, \xi}+j_{22} c_{i} P_{i, \eta}+j_{11} s_{i} P_{i, \xi}+j_{12} s_{i} P_{i, \eta}
\end{array}\right)
$$

\section{Etape 4 : matrice $B_{c \beta}$}

La matrice $B_{c \beta}(2 \times 12)$ relie les efforts tranchants aux déplacements de flexion.

$B_{c \beta}$ est définie par la relation [42] :

$$
B_{c \beta}=\overline{H_{f}} P_{f \beta}
$$

où $\overline{H_{f}}$ est donnée par l'expression [43]:

$$
\overline{H_{f}}=H_{f}\left(\begin{array}{cccccc}
1 & \frac{1-v}{2} & 0 & 0 & 0 & \frac{1+v}{2} \\
0 & 0 & \frac{1+v}{2} & \frac{1-v}{2} & 1 & 0
\end{array}\right)
$$

et où $\quad P_{f \beta}$ est définie par blocs : $P_{f \beta}=\left[\begin{array}{lllll}{\left[P_{f \beta 1}\right]} & {\left[P_{f \beta 2}\right]} & {\left[P_{f \beta 3}\right]} & {\left[P_{f \beta 4}\right]}\end{array}\right]$ avec des blocs de la forme [44] :

$$
\begin{aligned}
& P_{f \beta i}=\left(\begin{array}{cc}
0 & j_{11}^{2} N_{i, \xi^{2}}+2 j_{11} j_{12} N_{i, \xi}+j_{12}^{2} N_{i, \eta^{2}} \\
0 & j_{21}^{2} N_{i, \xi^{2}}+2 j_{21} j_{22} N_{i, \xi \eta}+j_{22}^{2} N_{i, \eta^{2}} \\
0 & j_{11} j_{21} N_{i, \xi^{2}}+\left(j_{11} j_{22}+j_{12} j_{21}\right) N_{i, \xi \eta}+j_{12} j_{22} N_{i, \eta^{2}} \\
0 & 0 \\
0 & 0 \\
0 & 0
\end{array}\right. \\
& \begin{array}{c}
0 \\
0 \\
0 \\
j_{11}^{2} N_{i, \xi^{2}}+2 j_{11} j_{12} N_{i, \xi \eta}+j_{12}^{2} N_{i, \eta^{2}} \\
j_{21}^{2} N_{i, \xi^{2}}+2 j_{21} j_{22} N_{i, \xi \eta}+j_{22}^{2} N_{i, \eta^{2}} \\
j_{11} j_{21} N_{i, \xi^{2}}+\left(j_{11} j_{22}+j_{12} j_{21}\right) N_{i, \xi \eta}+j_{12} j_{22} N_{i, \eta^{2}}
\end{array} \mid
\end{aligned}
$$

Etape 5 : matrice $B_{c \alpha}$

La matrice $B_{c \alpha}(2 \times 4)$ relie les efforts tranchants aux paramètres $\alpha_{k}$. 
$B_{c \alpha}$ est définie par la relation [45] :

$B_{c \alpha}=\overline{H_{f}} T_{2} T_{\alpha}$

$T_{2} \quad$ est définie par blocs : $T_{2}=\left(\begin{array}{ll}{\left[t_{2}\right]} & {[0]} \\ {[0]} & {\left[t_{2}\right]}\end{array}\right)$

avec le bloc $t_{2}$ de la forme [46]:

$$
\begin{aligned}
t_{2} & =\left(\begin{array}{ccc}
j_{11}^{2} & j_{12}^{2} & 2 j_{11} j_{12} \\
j_{21}^{2} & j_{22}^{2} & 2 j_{21} j_{22} \\
j_{11} j_{21} & j_{12} j_{22} & j_{11} j_{22}+j_{12} j_{21}
\end{array}\right) \\
T_{\alpha} & \text { est définie par blocs : } T_{\alpha}=\left[\begin{array}{llll}
{\left[T_{\alpha 1}\right]} & {\left[T_{\alpha 2}\right]} & {\left[T_{\alpha 3}\right]} & {\left[T_{\alpha 4}\right]}
\end{array}\right]
\end{aligned}
$$

avec le bloc $T_{\alpha i}$ de la forme [47] :

$$
T_{\alpha i}=\left|\begin{array}{c}
c_{i} P_{i, \xi^{2}} \\
c_{i} P_{i, \eta^{2}} \\
c_{i} P_{i, \xi \eta} \\
s_{i} P_{i, \xi^{2}} \\
s_{i} P_{i, \eta^{2}} \\
s_{i} P_{i, \xi \eta}
\end{array}\right|
$$

\section{Etape 6 : matrice $A_{\beta}$}

La matrice $A_{\beta}$ relie les paramètres $\alpha_{k}$ aux déplacements de flexion par la relation matricielle [48] :

$$
\left\{\begin{array}{c}
\alpha_{1} \\
\alpha_{2} \\
\alpha_{3} \\
\alpha_{4}
\end{array}\right)=A_{\beta}\left(\begin{array}{c}
w^{1} \\
\beta_{x}^{1} \\
\beta_{y}^{1} \\
\vdots \\
w^{4} \\
\beta_{x}^{4} \\
\beta_{y}^{4}
\end{array}\right\}
$$

$A_{\beta}$ est définie par la relation [49]:

$$
A_{\beta}=A_{\alpha}^{-1} A_{w}
$$

$A_{\alpha} \quad$ est donnée par l'expression matricielle [50] : 


$$
\begin{aligned}
A_{\alpha}= & \frac{2}{3}\left(\begin{array}{cccc}
L_{1} & 0 & 0 & 0 \\
0 & L_{2} & 0 & 0 \\
0 & 0 & L_{3} & 0 \\
0 & 0 & 0 & L_{4}
\end{array}\right) \\
& -\frac{1}{H_{c t}}\left(\begin{array}{cccccccc}
L_{1} c_{1} & L_{1} S_{1} & 0 & 0 & 0 & 0 & 0 & 0 \\
0 & 0 & L_{2} c_{2} & L_{2} S_{2} & 0 & 0 & 0 & 0 \\
0 & 0 & 0 & 0 & L_{3} c_{3} & L_{3} s_{3} & 0 & 0 \\
0 & 0 & 0 & 0 & 0 & 0 & L_{4} c_{4} & L_{4} S_{4}
\end{array}\right) \overline{B_{c \alpha}}
\end{aligned}
$$

où $H_{c t}$ est donnée par l'expression [51]:

$$
H_{c t}=\frac{5}{6} \frac{E h}{2(1+v)}
$$

et où $\quad \overline{B_{c \alpha}} \quad$ est définie par blocs : $\overline{B_{c \alpha}}=\left(\begin{array}{l}{\left[B_{c \alpha 1}\right]} \\ {\left[B_{c \alpha 2}\right]} \\ {\left[B_{c \alpha 3}\right]} \\ {\left[B_{c \alpha 4}\right]}\end{array}\right)$

avec des blocs de la forme [52] :

$$
B_{c \alpha i}=\frac{1}{L_{i}} \int_{\left[0, L_{i}\right]} B_{c \alpha} d s
$$

On évalue l'intégrale par une quadrature de Gauss à 2 points (d'abscisses $\pm \frac{1}{\sqrt{3}}$ dans l'élément de référence $[-1,1]$ et de poids $\left.\frac{1}{2}\right)$ notés $P G_{i l}$ et $P G_{i 2}$ dans l'expression [53]:

$$
\frac{1}{L_{i}} \int_{\left[0, L_{i}\right]} B_{c \alpha} d s \simeq \frac{1}{2}\left[\overline{H_{f}} T_{2}\left(P G_{i l}\right) T_{\alpha}\left(P G_{i 1}\right)+\overline{H_{f}} T_{2}\left(P G_{i 2}\right) T_{\alpha}\left(P G_{i 2}\right)\right]
$$

$A_{w}$ est donnée par l'expression matricielle [54] :

$$
A_{w}=-\frac{1}{2}\left(\begin{array}{ccccccccc}
-2 & L_{1} c_{1} & L_{1} s_{1} & 2 & L_{1} c_{1} & L_{1} s_{1} & 0 & 0 & 0 \\
0 & 0 & 0 & -2 & L_{2} c_{2} & L_{2} s_{2} & 2 & L_{2} c_{2} & L_{2} s_{2} \\
0 & 0 & 0 & 0 & 0 & 0 & -2 & L_{3} c_{3} & L_{3} s_{3} \\
2 & L_{4} c_{4} & L_{4} s_{4} & 0 & 0 & 0 & 0 & 0 & 0 \\
& 0 & 0 & 0 \\
0 & 0 & 0 \\
2 & L_{3} c_{3} & L_{3} s_{3} \\
-2 & L_{4} c_{4} & L_{4} s_{4}
\end{array}\right)
$$




$$
+\frac{1}{H_{c t}}\left(\begin{array}{cccccccc}
L_{1} c_{1} & L_{1} s_{1} & 0 & 0 & 0 & 0 & 0 & 0 \\
0 & 0 & L_{2} c_{2} & L_{2} s_{2} & 0 & 0 & 0 & 0 \\
0 & 0 & 0 & 0 & L_{3} c_{3} & L_{3} s_{3} & 0 & 0 \\
0 & 0 & 0 & 0 & 0 & 0 & L_{4} c_{4} & L_{4} s_{4}
\end{array}\right) \overline{B_{c \beta}}
$$

$\overline{B_{c \beta}} \quad$ est définie par blocs : $\overline{B_{c \beta}}=\left(\begin{array}{l}{\left[B_{c \beta 1}\right]} \\ {\left[B_{c \beta 2}\right]} \\ {\left[B_{c \beta 3}\right]} \\ {\left[B_{c \beta 4}\right]}\end{array}\right)$

avec des blocs de la forme [55]:

$$
B_{c \beta i}=\frac{1}{L_{i}} \int_{\left[0, L_{i}\right]} B_{c \beta} d s
$$

L'intégration est menée comme pour $B_{c \alpha i}$ et donnée par l'expression [56] :

$$
B_{c \beta i}=\frac{1}{L_{k}} \int_{\left[0, L_{i}\right]} B_{c \beta} d s \simeq \frac{1}{2}\left[\overline{H_{f}} P_{f \beta}\left(P G_{i l}\right)+\overline{H_{f}} P_{f \beta}\left(P G_{i 2}\right)\right]
$$

\begin{tabular}{|c|c|c|c|c|}
\hline \multirow{3}{*}{$\begin{array}{c}\text { Point d'étude } \\
\text { B }\end{array}$} & \multicolumn{2}{|c|}{ Pont droit } & \multicolumn{2}{|c|}{ Pont de biais modéré } \\
\hline & $x=24,45$ & $y=3,85$ & $x=27,34$ & $y=4,08$ \\
\hline & $\begin{array}{l}\text { Intégration } \\
\text { influences }\end{array}$ & $\begin{array}{l}\text { Calcul } \\
\text { direct }\end{array}$ & $\begin{array}{l}\text { Intégration } \\
\text { influences }\end{array}$ & $\begin{array}{l}\text { Calcul } \\
\text { direct }\end{array}$ \\
\hline $\begin{array}{c}M_{x} \\
(\mathrm{kN} \cdot \mathrm{m} / \mathrm{m})\end{array}$ & 19,6172 & 19,6172 & 13,6612 & 13,6612 \\
\hline $\begin{array}{c}M_{y} \\
(\mathrm{kN} \cdot \mathrm{m} / \mathrm{m})\end{array}$ & 0,8049 & 0,8049 & 1,1162 & 1,1162 \\
\hline $\begin{array}{c}M_{x y} \\
(\mathrm{kN} \cdot \mathrm{m} / \mathrm{m})\end{array}$ & $-0,0527$ & $-0,0527$ & $-3,9088$ & $-3,9088$ \\
\hline $\begin{array}{c}T_{x} \\
(\mathrm{kN} / \mathrm{m})\end{array}$ & 0,1304 & 0,1304 & $-0,4795$ & $-0,4796$ \\
\hline $\begin{array}{c}T_{y} \\
(\mathrm{kN} / \mathrm{m})\end{array}$ & $-0,3786$ & $-0,3786$ & $-0,5953$ & $-0,5952$ \\
\hline
\end{tabular}

\section{Annexe 2 : résultats pour les points d'étude $\mathrm{B}$ à $\mathrm{E}$}

Tableau 3a. Comparaison du calcul direct et de l'intégration des influences pour le point d'étude $B$ : chargement de toute la surface 
524 Revue européenne de mécanique numérique. Volume $17-\mathrm{n}^{\circ}$ 4/2008

\begin{tabular}{|c|c|c|c|c|}
\hline \multirow{2}{*}{$\begin{array}{c}\text { Point d'étude } \\
\text { B }\end{array}$} & \multicolumn{2}{|c|}{ Pont droit } & \multicolumn{2}{c|}{$\begin{array}{c}\text { Pont de biais modéré } \\
x=27,45\end{array}$} \\
\cline { 2 - 5 } & $\begin{array}{c}\text { Intégration } \\
\text { influences }\end{array}$ & $\begin{array}{c}\text { Calcul } \\
\text { direct }\end{array}$ & $\begin{array}{c}\text { Intégration } \\
\text { influences }\end{array}$ & $\begin{array}{c}\text { Calcul } \\
\text { direct }\end{array}$ \\
\hline $\begin{array}{c}M_{x} \\
(\mathrm{kN} \cdot \mathrm{m} / \mathrm{m})\end{array}$ & 0,6409 & 0,6409 & 0,7491 & 0,7491 \\
\hline $\begin{array}{c}M_{y} \\
(\mathrm{kN} \cdot \mathrm{m} / \mathrm{m})\end{array}$ & 0,1518 & 0,1518 & 0,1462 & 0,1462 \\
\hline $\begin{array}{c}M_{x y} \\
(\mathrm{kN} \cdot \mathrm{m} / \mathrm{m})\end{array}$ & 0,0895 & 0,0895 & $-0,1900$ & $-0,1897$ \\
\hline $\begin{array}{c}T_{x} \\
(\mathrm{kN} / \mathrm{m})\end{array}$ & $-0,2748$ & $-0,2748$ & $-0,2415$ & $-0,2415$ \\
\hline $\begin{array}{c}T_{y} \\
(\mathrm{kN} / \mathrm{m})\end{array}$ & $-0,2726$ & $-0,2726$ & $-0,2127$ & $-0,2127$ \\
\hline
\end{tabular}

Tableau 3b. Comparaison du calcul direct et de l'intégration des influences pour le point d'étude $B$ : chargement d'une zone d'impact

\begin{tabular}{|c|c|c|c|c|}
\hline \multirow{3}{*}{$\begin{array}{c}\text { Point d'étude } \\
\text { C }\end{array}$} & \multicolumn{2}{|c|}{ Pont droit } & \multicolumn{2}{|c|}{ Pont de biais modéré } \\
\hline & $x=14,60$ & $y=3,85$ & $x=19,28$ & $y=4,08$ \\
\hline & $\begin{array}{l}\text { Intégration } \\
\text { influences }\end{array}$ & $\begin{array}{l}\text { Calcul } \\
\text { direct }\end{array}$ & $\begin{array}{l}\text { Intégration } \\
\text { influences }\end{array}$ & $\begin{array}{l}\text { Calcul } \\
\text { direct }\end{array}$ \\
\hline $\begin{array}{c}M_{x} \\
(\mathrm{kN} \cdot \mathrm{m} / \mathrm{m})\end{array}$ & $-39,7652$ & $-39,7729$ & 13,6612 & 13,6612 \\
\hline $\begin{array}{c}M_{y} \\
(\mathrm{kN} \cdot \mathrm{m} / \mathrm{m})\end{array}$ & $-15,8788$ & $-15,8867$ & 1,1162 & 1,1162 \\
\hline $\begin{array}{c}M_{x y} \\
(\mathrm{kN} \cdot \mathrm{m} / \mathrm{m})\end{array}$ & $-0,2318$ & $-0,2318$ & $-3,9088$ & $-3,9088$ \\
\hline $\begin{array}{c}T_{x} \\
(\mathrm{kN} / \mathrm{m})\end{array}$ & 0,3180 & 0,3180 & $-0,4795$ & $-0,4796$ \\
\hline $\begin{array}{c}T_{y} \\
(\mathrm{kN} / \mathrm{m})\end{array}$ & $-5,2487$ & $-5,2512$ & $-0,5953$ & $-0,5952$ \\
\hline
\end{tabular}

Tableau 4a. Comparaison du calcul direct et de l'intégration des influences pour le point d'étude $C$ : chargement de toute la surface 


\begin{tabular}{|c|c|c|c|c|}
\hline \multirow{3}{*}{$\begin{array}{c}\text { Point d'étude } \\
\text { C }\end{array}$} & & \multicolumn{2}{|c|}{ Pont de biais modéré } \\
\hline & \multicolumn{2}{|c|}{$x=14,60 \quad y=3,85$} & \multicolumn{2}{|c|}{$x=19,28 \quad y=4,08$} \\
\hline & $\begin{array}{c}\text { Intégration } \\
\text { influences }\end{array}$ & $\begin{array}{l}\text { Calcul } \\
\text { direct }\end{array}$ & $\begin{array}{l}\text { Intégration } \\
\text { influences }\end{array}$ & $\begin{array}{l}\text { Calcul } \\
\text { direct }\end{array}$ \\
\hline $\begin{array}{c}M_{x} \\
(\mathrm{kN} \cdot \mathrm{m} / \mathrm{m})\end{array}$ & $-0,2790$ & $-0,2792$ & $-0,3077$ & $-0,3078$ \\
\hline $\begin{array}{c}M_{y} \\
(\mathrm{kN} \cdot \mathrm{m} / \mathrm{m})\end{array}$ & $-0,2777$ & $-0,2779$ & $-0,2985$ & $-0,2985$ \\
\hline $\begin{array}{c}M_{x y} \\
(\mathrm{kN} \cdot \mathrm{m} / \mathrm{m})\end{array}$ & 0,1385 & 0,1385 & 0,1222 & 0,1222 \\
\hline $\begin{array}{c}T_{x} \\
(\mathrm{kN} / \mathrm{m})\end{array}$ & $-0,2791$ & $-0,2791$ & $-0,2342$ & $-0,2342$ \\
\hline $\begin{array}{c}T_{y} \\
(\mathrm{kN} / \mathrm{m})\end{array}$ & $-0,3941$ & $-0,3941$ & $-0,2178$ & $-0,2177$ \\
\hline
\end{tabular}

Tableau 4b. Comparaison du calcul direct et de l'intégration des influences pour le point d'étude C : chargement d'une zone d'impact

\begin{tabular}{|c|c|c|c|c|}
\hline \multirow{2}{*}{$\begin{array}{c}\text { Point d'étude } \\
\text { D }\end{array}$} & \multicolumn{2}{|c|}{ Pont droit } & \multicolumn{2}{c|}{$\begin{array}{c}\text { Pont de biais modéré } \\
x=18,09\end{array}$} \\
\cline { 2 - 5 } & $\begin{array}{c}\text { Intégration } \\
\text { influences }\end{array}$ & $\begin{array}{c}\text { Calcul } \\
\text { direct }\end{array}$ & $\begin{array}{c}\text { Intégration } \\
\text { influences }\end{array}$ & $\begin{array}{c}\text { Calcul } \\
\text { direct }\end{array}$ \\
\hline $\begin{array}{c}M_{x} \\
(\mathrm{kN} . \mathrm{m} / \mathrm{m})\end{array}$ & $-20,1761$ & $-20,1761$ & $-14,3340$ & $-14,3342$ \\
\hline $\begin{array}{c}M_{y} \\
(\mathrm{kN} . \mathrm{m} / \mathrm{m})\end{array}$ & $-2,3175$ & $-2,3175$ & $-2,5459$ & $-2,5459$ \\
\hline $\begin{array}{c}M_{x y} \\
(\mathrm{kN} . \mathrm{m} / \mathrm{m})\end{array}$ & 0,2710 & 0,2710 & 3,2923 & 3,2920 \\
\hline $\begin{array}{c}T_{x} \\
(\mathrm{kN} / \mathrm{m})\end{array}$ & $-8,2795$ & $-8,2795$ & $-5,6523$ & $-5,6529$ \\
\hline $\begin{array}{c}T_{y} \\
(\mathrm{kN} / \mathrm{m})\end{array}$ & 0,6523 & 0,6523 & 0,3551 & 0,3565 \\
\hline
\end{tabular}

Tableau 5a. Comparaison du calcul direct et de l'intégration des influences pour le point d'étude D : chargement de toute la surface 
526 Revue européenne de mécanique numérique. Volume $17-\mathrm{n}^{\circ}$ 4/2008

\begin{tabular}{|c|c|c|c|c|}
\hline \multirow{2}{*}{$\begin{array}{c}\text { Point d'étude } \\
\text { D }\end{array}$} & \multicolumn{2}{|c|}{ Pont droit } & \multicolumn{2}{c|}{$\begin{array}{c}\text { Pont de biais modéré } \\
x=18,41\end{array}$} \\
\cline { 2 - 5 } & $\begin{array}{c}\text { Intégration } \\
\text { influences }\end{array}$ & $\begin{array}{c}\text { Calcul } \\
\text { direct }\end{array}$ & $\begin{array}{c}\text { Intégration } \\
\text { influences }\end{array}$ & $\begin{array}{c}\text { Calcul } \\
\text { direct }\end{array}$ \\
\hline $\begin{array}{c}M_{x} \\
(\mathrm{kN} \cdot \mathrm{m} / \mathrm{m})\end{array}$ & 0,2040 & 0,2040 & 0,2454 & 0,2454 \\
\hline $\begin{array}{c}M_{y} \\
(\mathrm{kN} \cdot \mathrm{m} / \mathrm{m})\end{array}$ & 0,0799 & 0,0799 & 0,0829 & 0,0829 \\
\hline $\begin{array}{c}M_{x y} \\
(\mathrm{kN} \cdot \mathrm{m} / \mathrm{m})\end{array}$ & 0,1914 & 0,1914 & 0,1663 & 0,1663 \\
\hline $\begin{array}{c}T_{x} \\
(\mathrm{kN} / \mathrm{m})\end{array}$ & $-0,4686$ & $-0,4686$ & $-0,3422$ & $-0,3422$ \\
\hline $\begin{array}{c}T_{y} \\
(\mathrm{kN} / \mathrm{m})\end{array}$ & $-0,2757$ & $-0,2757$ & $-0,3018$ & $-0,2018$ \\
\hline
\end{tabular}

Tableau 5b. Comparaison du calcul direct et de l'intégration des influences pour le point d'étude D : chargement d'une zone d'impact

\begin{tabular}{|c|c|c|c|c|}
\hline \multirow{3}{*}{$\begin{array}{c}\text { Point d'étude } \\
\text { E }\end{array}$} & \multicolumn{2}{|c|}{ Pont droit } & \multicolumn{2}{|c|}{ Pont de biais modéré } \\
\hline & $x=15,79$ & $y=3,85$ & $x=20,47$ & $y=4,08$ \\
\hline & $\begin{array}{l}\text { Intégration } \\
\text { influences }\end{array}$ & $\begin{array}{l}\text { Calcul } \\
\text { direct }\end{array}$ & $\begin{array}{l}\text { Intégration } \\
\text { influences }\end{array}$ & $\begin{array}{l}\text { Calcul } \\
\text { direct }\end{array}$ \\
\hline $\begin{array}{c}M_{x} \\
(\mathrm{kN} \cdot \mathrm{m} / \mathrm{m})\end{array}$ & $-19,1637$ & $-19,1634$ & $-6,1689$ & $-6,1690$ \\
\hline $\begin{array}{c}M_{y} \\
(\mathrm{kN} \cdot \mathrm{m} / \mathrm{m})\end{array}$ & $-2,3018$ & $-2,3017$ & $-2,6124$ & $-2,6123$ \\
\hline $\begin{array}{c}M_{x y} \\
(\mathrm{kN} \cdot \mathrm{m} / \mathrm{m})\end{array}$ & $-0,7328$ & $-0,7328$ & $-3,9170$ & $-3,9172$ \\
\hline $\begin{array}{c}T_{x} \\
(\mathrm{kN} / \mathrm{m})\end{array}$ & 8,9661 & 8,9662 & 10,8948 & 10,8947 \\
\hline $\begin{array}{c}T_{y} \\
(\mathrm{kN} / \mathrm{m})\end{array}$ & 0,6471 & 0,6471 & $-1,6833$ & $-1,6831$ \\
\hline
\end{tabular}

Tableau 6a. Comparaison du calcul direct et de l'intégration des influences pour le point d'étude E : chargement de toute la surface 


\begin{tabular}{|c|c|c|c|c|}
\hline \multirow{2}{*}{$\begin{array}{c}\text { Point d'étude } \\
\mathrm{E}\end{array}$} & \multicolumn{2}{|c|}{ Pont droit } & \multicolumn{2}{c|}{$\begin{array}{c}\text { Pont de biais modéré } \\
x=20,47\end{array}$} \\
\cline { 2 - 5 } & $\begin{array}{c}\text { Intégration } \\
\text { influences }\end{array}$ & $\begin{array}{c}\text { Calcul } \\
\text { direct }\end{array}$ & $\begin{array}{c}\text { Intégration } \\
\text { influences }\end{array}$ & $\begin{array}{c}\text { Calcul } \\
\text { direct }\end{array}$ \\
\hline $\begin{array}{c}M_{x} \\
(\mathrm{kN} . \mathrm{m} / \mathrm{m})\end{array}$ & 0,1898 & 0,1898 & 0,2295 & 0,2295 \\
\hline $\begin{array}{c}M_{y} \\
(\mathrm{kN} . \mathrm{m} / \mathrm{m})\end{array}$ & 0,0787 & 0,0787 & 0,0661 & 0,0661 \\
\hline $\begin{array}{c}M_{x y} \\
(\mathrm{kN} . \mathrm{m} / \mathrm{m})\end{array}$ & 0,8319 & 0,8319 & $-0,2476$ & $-0,2476$ \\
\hline $\begin{array}{c}T_{x} \\
(\mathrm{kN} / \mathrm{m})\end{array}$ & $-0,0712$ & 0,0712 & $-0,0107$ & $-0,0107$ \\
\hline $\begin{array}{c}T_{y} \\
(\mathrm{kN} / \mathrm{m})\end{array}$ & $-0,2741$ & $-0,2741$ & $-0,2599$ & $-0,2599$ \\
\hline
\end{tabular}

Tableau 6b. Comparaison du calcul direct et de l'intégration des influences pour le point d'étude E : chargement d'une zone d'impact 
